\title{
Zusammenarbeit in geregelten Bahnen
}

Erfolgreiche Zusammenarbeit (Collaboration) ist ein wichtiger Erfolgsfaktor in jedem Umfeld, und es bedarf geeigneter Vorgaben, damit die Zusammenarbeit in geregelten Bahnen verläuft. DITA bietet dazu ab Version 1.2 gute Unterstützung.

Mit Constraints kann die Syntax ohne Spezialisierung eingeschränkt werden. Auf diese Weise können Konventionen in einem Projektumfeld für ein Team eindeutiger festgelegt werden. So können optionale Elemente entfernt oder obligatorisch gesetzt und die Reihenfolge von Elementen vorgegeben werden.

Eine vielversprechende Möglichkeit, klare Vorgaben zu machen, bieten Subject Schemas. Sie erlauben für ein konkretes Umfeld die Festlegung von spezifischen Attributwerten und Bezeichnungen für Metadaten, ohne in die DTDs eingreifen zu müssen.

Ergänzungen für die Begriffswelt ermöglichen die Definition von Terminologie bis hin zu kompletten Taxonomien. Damit kann die Grundlage für semantische Web-Funktionen geschaffen werden: systematisch, standardisiert und integriert in die Quellinhalte. 